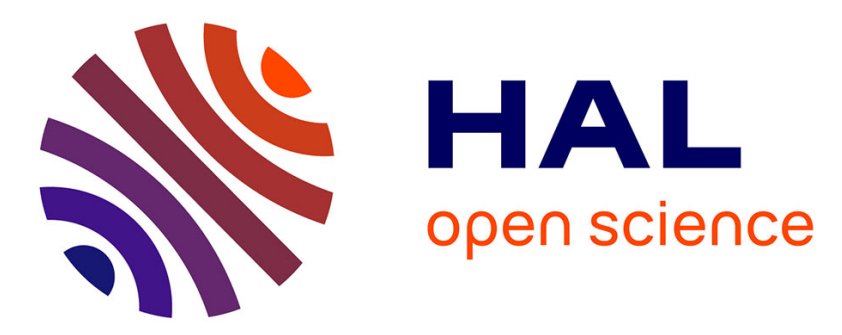

\title{
A spatiotemporal statistical atlas of motion for the quantification of abnormal myocardial tissue velocities
}

Nicolas Duchateau, Mathieu de Craene, Gemma Piella, Etelvino Silva, Adelina Doltra, Marta Sitges, Bart Bijnens, Alejandro Frangi

\section{To cite this version:}

Nicolas Duchateau, Mathieu de Craene, Gemma Piella, Etelvino Silva, Adelina Doltra, et al.. A spatiotemporal statistical atlas of motion for the quantification of abnormal myocardial tissue velocities. Medical Image Analysis, 2011, 15 (3), pp.316-328. 10.1016/j.media.2010.12.006 . hal-02282430

\section{HAL Id: hal-02282430 \\ https://hal.science/hal-02282430}

Submitted on 11 Sep 2019

HAL is a multi-disciplinary open access archive for the deposit and dissemination of scientific research documents, whether they are published or not. The documents may come from teaching and research institutions in France or abroad, or from public or private research centers.
L'archive ouverte pluridisciplinaire HAL, est destinée au dépôt et à la diffusion de documents scientifiques de niveau recherche, publiés ou non, émanant des établissements d'enseignement et de recherche français ou étrangers, des laboratoires publics ou privés. 


\title{
Preprint version accepted to appear in Medical Image Analysis. Final version of this paper will be available from http://www.sciencedirect.com/science/journal/13618415
}

\section{A Spatiotemporal Statistical Atlas of Motion for the Quantification of Abnormal Myocardial Tissue Velocities}

\author{
Nicolas Duchateau ${ }^{\mathrm{a}, \mathrm{b}}$, Mathieu De Craene ${ }^{\mathrm{a}, \mathrm{b}}$, Gemma Piella $^{\mathrm{a}, \mathrm{b}}$, Etelvino Silva $^{\mathrm{c}}$, Adelina Doltra $^{\mathrm{c}}$, \\ Marta Sitges ${ }^{\mathrm{c}}$, Bart H. Bijnens ${ }^{\mathrm{d}}$, Alejandro F. Frangi ${ }^{\mathrm{a}, \mathrm{b}, \mathrm{d}, *}$ \\ ${ }^{a}$ Center for Computational Imaging \& Simulation Technologies in Biomedicine; Information \& Communication Technologies \\ Department, Universitat Pompeu Fabra, Barcelona, Spain \\ ${ }^{b}$ Centro de Investigación Biomédica en Red en Bioingeniería, Biomateriales y Nanomedicina (CIBER-BBN) \\ ${ }^{c}$ Hospital Clínic; IDIBAPS; Universitat de Barcelona, Spain \\ ${ }^{d}$ Institució Catalana de Recerca $i$ Estudis Avançats (ICREA)
}

\begin{abstract}
In this paper, we present a new method for the automatic comparison of myocardial motion patterns and the characterization of their degree of abnormality, based on a statistical atlas of motion built from a reference healthy population. Our main contribution is the computation of atlas-based indexes that quantify the abnormality in the motion of a given subject against a reference population, at every location in time and space. The critical computational cost inherent to the construction of an atlas is highly reduced by the definition of myocardial velocities under a small displacements hypothesis. The indexes we propose are of notable interest for the assessment of anomalies in cardiac mobility and synchronicity when applied, for instance, to candidate selection for cardiac resynchronization therapy (CRT). We built an atlas of normality using 2D ultrasound cardiac sequences from 21 healthy volunteers, to which we compared 14 CRT patients with left ventricular dyssynchrony (LVDYS). We illustrate the potential of our approach in characterizing septal flash, a specific motion pattern related to LVDYS and recently introduced as a very good predictor of response to CRT.
\end{abstract}

Keywords: Cardiac atlas, motion analysis, myocardial motion, CRT

\section{Introduction}

\subsection{Patient selection for CRT}

Cardiac resynchronization therapy (CRT) has proved its benefits over the last few years for the treatment of patients with heart failure and evidence of ventricular conduction delays (Cleland et al., 2005). The objective of CRT is to restore the coordination in the motion of the cardiac chambers, leading to notable improvements in cardiac function and reverse remodeling (St John Sutton et al., 2003). However, with current selection criteria, the therapy fails to improve patient condition for approximately $30 \%$ of the subjects (Stellbrink

*c/Tànger 122-140, E08018 Barcelona, Spain. Tel: +34 93-542-1451, Fax: +34 93-542-1445

Email addresses: nicolas.duchateau@upf .edu

(Nicolas Duchateau), alejandro.frangi@upf.edu

(Alejandro F. Frangi) et al., 2004). The main current clinical challenge behind CRT is therefore the understanding of the physiological mechanisms conditioning positive or negative response.

In recent years, a large number of studies focused on the computation of quantitative indexes for cardiac dyssynchrony, with the underlying objective of predicting CRT response (Hawkins et al., 2006). The indexes proposed in the literature are mostly based on direct comparisons of temporal measurements (QRS duration and "time-to-peak" measures) (Bax et al., 2004), but they remain suboptimal as discussed in Voigt (2009) and Fornwalt et al. (2009) (poor reproducibility and simplification of the complex explication of CRT response to single observations of dyssychrony). The lack of consensus about indexes able to accurately predict CRT response proves that generic indexes that try to capture dyssynchrony with limited reference to patho- 


\section{Preprint version accepted to appear in Medical Image Analysis. Final version of this paper will be available from http://www.sciencedirect.com/science/journal/13618415}

physiology fail in the CRT context (Fornwalt et al., 2009). In order to fundamentally improve the prognostic value of novel indexes it is crucial that they are inspired in a deep understanding of the pathophysiological mechanisms involved in electrical and mechanical dyssynchrony. Recently, Parsai et al. (2009b) proposed a classification of patients into specific etiologies of heart failure, and evaluated the response of each of these groups. Using this classification, one group showing a specific left ventricle (LV) dyssynchrony pattern called septal flash (SF) (Parsai et al., 2009a) demonstrated a very high response rate to CRT (Parsai et al., 2009b).

\subsection{Quantifying abnormality in cardiac motion}

The SF pattern has been characterized in Parsai et al. (2009a,b), using M-mode echocardiography. The protocol presented allows quantitative assessment of the SF (presence, timing and maximal excursion). More automatic methods focusing on abnormal patterns associated with dyssynchrony have also been proposed, using speckle tracking strain analysis from 2D ultrasound (2D US) (Delgado et al., 2008), volume curves analysis from 3D US (Sonne et al., 2009), and circumferential shortening indexes from tagged magnetic resonance (t-MRI) images (Rutz et al., 2009). However, for such methods, the analysis is only performed in a limited set of points that are observer-defined or only representative of specific heart segments. The definition of these points is still highly subjective and patient-dependent. Thus, the variability in their localization limits the relevance of defining statistical indexes at such locations. In methods derived from recent advances in computational anatomy (Grenander and Miller, 1998), and particularly when using statistical atlases (Young and Frangi, 2009), patient data is normalized to a common anatomical reference, so that there is no need to define specific comparison points between patients. Such methods represent a promising alternative to compute relevant statistical indexes for the whole cardiac anatomy.

In our study, we aim at characterizing one aspect of the cardiac function, namely, motion throughout the heart cycle. Hence we rely on dynamic atlases, taking advantage of previous works on statistical atlases of motion and deformation initiated in Rao et al. (2004), Chandrashekara et al. (2005) and Rougon et al. (2004). We can distinguish three steps in the process of building such a statistical atlas:
Extracting motion from cardiac sequences. (Ledesma-Carbayo et al., 2005; Chandrashekara et al., 2004; Petitjean et al., 2004). In Khan and Beg (2008) and De Craene et al. (2009, 2010) the tracking along longitudinal datasets is combined with the diffeomorphic framework (Trouvé, 1998), particularly suitable when handling cardiac sequences, since it preserves the topology and the orientation of anatomical structures.

Normalizing the different sequences to a reference anatomy. A pipeline adapted to cardiac studies was used in Perperidis et al. (2005) and Peyrat et al. (2009). In Qiu et al. (2009) and Durrleman et al. (2009), the synchronization of longitudinal datasets is combined with the use of diffeomorphic paths to compare the evolution of shapes along different sequences. These approaches still need to prove their feasibility (robustness, computational cost) when applied to real data, especially when the topology of the structure of interest is not preserved along the sequence, due to the presence of noise.

Computing statistics on motion fields. To preserve the diffeomorphic properties of the computed vector fields, the use of log-Euclidean metrics is recommended when computing statistics, as summarized in Pennec and Fillard (2010). Abnormality assessment at every desired point of the anatomy requires the use of voxel-based morphometry tools (VBM) (Ashburner and Friston, 2000), for which an overview of some applications in brain morphometry can be found in Ashburner et al. (2003). Extending VBM tools to multivariate statistics (Worsley et al., 2004) allows to handle statistics on vector fields, similarly to the works that have been proposed for tensor fields (Leporé et al., 2008; Commowick et al., 2008).

\subsection{Proposed approach}

In this paper, we propose a complete and flexible pipeline for the construction of an atlas of motion based on these three construction steps, which were kept as simple as possible to avoid computational burden. Thus, each of these steps can further be improved using a more elaborated technique, provided this guarantees a noticeable improvement in the identification of abnormal motion patterns.

Cardiac anatomy is tracked using the chaining of diffeomorphic paths between pairs of consecutive frames. We take advantage of the high temporal 


\section{Preprint version accepted to appear in Medical Image Analysis. Final version of this paper will be available from http://www.sciencedirect.com/science/journal/13618415}

resolution of 2D US to work under a small displacements hypothesis. The use of small displacements reduces the computational complexity of estimating velocities over the whole continuous timescale, and allows direct computation of classical statistics on the velocity fields without the need of the logEuclidean framework.

The atlas is then used for the comparison of individuals to a healthy population, both represented by myocardial velocities, using abnormality indexes available at any location $(\mathbf{x}, t)$. One interesting feature of such indexes is that they intrinsically perform a comparison to normality. This contrasts with the indexes generally used for CRT, which usually measure one clinical parameter, and subsequently compare the ranges obtained for populations of healthy and diseased subjects to define an optimal separation threshold.

The method is applied to the analysis of a population of CRT patients with left ventricular dyssynchrony, looking for the presence of SF. A first preliminary version of this work was presented in Duchateau et al. (2009), in which we illustrated the feasibility of such an approach for assessing abnormality on a reduced number of patients.

\section{Computation of myocardial velocities}

\subsection{Intra-series registration}

In the following sections we will denote $\mathcal{S}=$ $\left\{\mathbf{S}\left(t_{0}\right), \ldots, \mathbf{S}\left(t_{i}\right), \ldots, \mathbf{S}\left(t_{N-1}\right)\right\}$ the temporal series of $2 \mathrm{D}$ images for one given patient, which contains $N$ images taken at time-points $t_{i}$. To track the anatomy along cardiac cycles, pairwise registration between consecutive frames provides a sequence of transformations $\boldsymbol{\varphi}_{t_{i}, t_{i+1}}: \mathbf{x} \mapsto \mathbf{x}^{\prime}$ for each series, which map any point $\mathbf{x}$ of image $\mathbf{S}\left(t_{i}\right)$ to its corresponding point $\mathbf{x}^{\prime}$ in the following frame $\mathbf{S}\left(t_{i+1}\right)$. Our non-rigid registration uses the diffeomorphic free-form deformation (FFD) method (Rueckert et al., 2006), which is made multi-resolution to improve its robustness to the position and spacing of control points. We used spacings of 64,32 and $16 \mathrm{~mm}$, and mutual information as matching term. The L-BFGS-B algorithm (Byrd et al., 1995) was chosen as optimizer for the registration procedure.

\subsection{Small displacement hypothesis and definition of velocities}

As explained in Arsigny et al. (2006), a diffeomorphism can be represented as the flow of a stationary velocity field uniquely defined by its logarithm. In compliance with the registration scheme we use, velocities can be written as piecewise stationary, using:

$$
\mathbf{v}\left(\varphi_{t_{i}, t}(\mathbf{x}), t\right)=\mathbf{v}\left(\mathbf{x}, t_{i}\right),
$$

where $t_{i}$ is the closest time-point that precedes $t$ at which the series $\mathcal{S}$ is defined, and $\boldsymbol{\varphi}_{t_{i}, t}(\mathbf{x})$ is the estimated position at time $t$ of the anatomical point that was at $\mathbf{x}$ at time $t_{i}$.

If the displacements are small, the logarithm of a transformation $\log \left(\boldsymbol{\varphi}_{t_{i}, t_{i+1}}\right)$ can be approximated at the first order by its corresponding displacement field $\boldsymbol{\varphi}_{t_{i}, t_{i+1}}-\mathbf{I}$ (where $\mathbf{I}$ is the identity). Velocities are directly obtained at the discrete time-points $t_{i}$ where the data is defined using:

$$
\begin{aligned}
\left(t_{i+1}-t_{i}\right) \cdot \mathbf{v}\left(., t_{i}\right) & =\log \left(\boldsymbol{\varphi}_{t_{i}, t_{i+1}}\right) \\
& \approx \boldsymbol{\varphi}_{t_{i}, t_{i+1}}-\mathbf{I} .
\end{aligned}
$$

These equations are coherent with the classical definition of velocities in mechanics, that is to say a displacement normalized by time.

The use of small displacements allows some additional simplifications in the computation of velocities at every time $t$, initially based on Eq. 1. First, $\boldsymbol{\varphi}_{t_{i}, t}$ can be estimated from $\boldsymbol{\varphi}_{t_{i}, t_{i+1}}$ using:

$$
\boldsymbol{\varphi}_{t_{i}, t}-\mathbf{I} \approx \frac{t-t_{i}}{t_{i+1}-t_{i}} \cdot\left(\boldsymbol{\varphi}_{t_{i}, t_{i+1}}-\mathbf{I}\right) .
$$

In a similar way, its inverse can be written as:

$$
\varphi_{t_{i}, t}^{-1}=\varphi_{t, t_{i}} \approx-\varphi_{t_{i}, t}
$$

This leads to the following simplified expressions for the velocities:

$$
\mathbf{v}(., t) \approx\left\{\begin{array}{lr}
\left(\boldsymbol{\varphi}_{t_{i}, t_{i+1}}-\mathbf{I}\right) /\left(t_{i+1}-t_{i}\right) & \text { if } t=t_{i} \\
\mathbf{v}\left(-\boldsymbol{\varphi}_{t_{i}, t}(.), t_{i}\right) & \text { otherwise }
\end{array}\right.
$$

With this formulation, orientation and invertibility are preserved at any point $(\mathbf{x}, t)$, i.e. trajectories are guaranteed to be diffeomorphic at any time $t$, as the log-exponential does with large displacements.

\subsection{Verifying the small displacements hypothesis on 2D US sequences}

We can reasonably assume that the displacements between consecutive frames are small. Such a choice is justified by the good temporal resolution 


\section{Preprint version accepted to appear in Medical Image Analysis. Final version of this paper will be available from http://www.sciencedirect.com/science/journal/13618415}

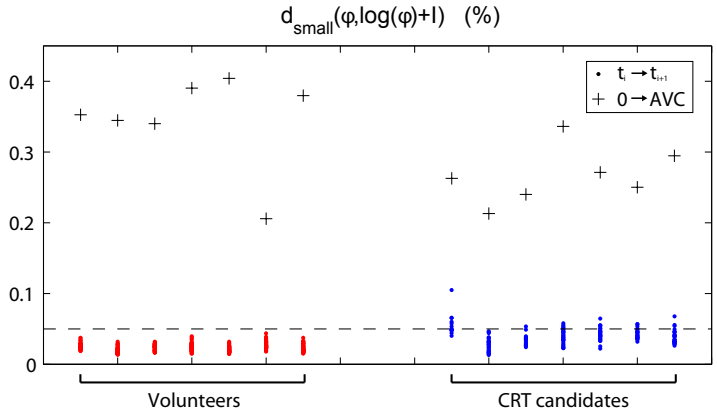

Figure 1: Distribution of the dissimilarity measure $d_{\text {small }}$ for all the mappings between consecutive frames of seven volunteers and seven CRT candidates with SF (blue and red dots). The same distance was computed for the mapping between the initial frame in the cycle and the frame at aortic valve closure (AVC), which produces larger displacements (crosses).

of 2D US imaging (around 60 frames/s [fps] for the healthy subjects and 30 fps for the CRT ones, details are in Sec. 4.1). We demonstrated the validity of this assumption by comparing the computed displacement fields to the logarithm of their relative transformations. We used

$d_{\text {small }}\left(\boldsymbol{\varphi}_{\mathbf{1}}, \boldsymbol{\varphi}_{\mathbf{2}}\right)=\frac{1}{\operatorname{card}(\boldsymbol{\Omega})} \cdot \sum_{\mathbf{x} \in \boldsymbol{\Omega}} \frac{\left|\boldsymbol{\varphi}_{\mathbf{2}} \circ \boldsymbol{\varphi}_{\mathbf{1}}^{-1}-\mathbf{I}\right|}{\left|\boldsymbol{\varphi}_{\mathbf{1}}-\mathbf{I}\right|}(\mathbf{x})$

as normalized dissimilarity measure between two transformations $\varphi_{\mathbf{1}}$ and $\boldsymbol{\varphi}_{\mathbf{2}}$, where $\boldsymbol{\Omega}$ is the image domain. Details about the computation of the logarithm and the inverse of the transformations $\boldsymbol{\varphi}_{t_{i}, t_{i+1}}$ are given in Arsigny et al. (2006).

This comparison is illustrated in Fig. 1 for seven healthy volunteers and seven CRT candidates with SF. The computation involved all the frames contained into one cardiac cycle. The distance is computed for the mappings between consecutive frames (dots), showing there is on average less than $5 \%$ difference between the computed displacement fields and the logarithm of their relative transformations. This confirms that the displacements can be considered as small, and that the velocities can therefore be computed using the simplified expression of Eq. 6. For comparison purposes, this computation was also done for the transformation mapping the initial frame in the cycle and the frame at end-systole (aortic valve closure event, defined in Sec. 3.1), resulting in larger displacements (crosses), and a distance $d_{\text {small }}$ between 20 and $40 \%$ difference.

\subsubsection{Small displacements and gain in computa- tional time}

The use of the small displacements hypothesis and the simplifications from Eq. 3, 4, and 5 allow much faster computations, which are particularly recommended in the context of building an atlas involving a large amount of data. Without the use of small displacements, computing velocities at times $t_{i}$ (Eq. 2) and $t$ (Eq. 1) requires 50 and 15 seconds respectively, using a Intel Core i7 $920(2.66 \mathrm{GHz}$ CPU, 6 GB RAM) computer. In comparison, the computational time is negligible when using the simplified expressions summarized in Eq. 6, since no logarithm nor inverse computation is required.

\section{Construction of the Atlas}

The registration steps previously explained provide velocity fields defined in the anatomy of each patient. Building an atlas requires bringing these fields to a common spatiotemporal coordinate system, so that a statistical representation of the data can be provided at every desired location $(\mathbf{x}, t)$.

In the following, we use $k$ to refer to the $k$-th sample patient, and we index variable names accordingly.

\subsection{Temporal synchronization}

The heart rate variability between patients changes the length of their respective cardiac cycles, as well as the synchronization of the different phases composing each cycle. Sequences may also differ in terms of trigger time and frame rate. Temporal synchronization will therefore consist in establishing correspondences between the cardiac events of the considered sequences and in bringing them to a normalized timescale.

Landmark-based piecewise linear warping is applied to the electrocardiogram (ECG) signals in order to map the sequences to a normalized timescale, as illustrated in Fig. 2. We use the following three landmarks:

- The onset of the QRS complex, which is located on the ECG using tools from the EchoPac software (GE Vingmed Ultrasound A.S., Horten, Norway).

- The aortic valve opening (AVO) and closure (AVC), which are determined using continuous wave Doppler imaging on the aortic valve. AVO 


\section{Preprint version accepted to appear in Medical Image Analysis. Final version of this paper will be available from http://www.sciencedirect.com/science/journal/13618415}

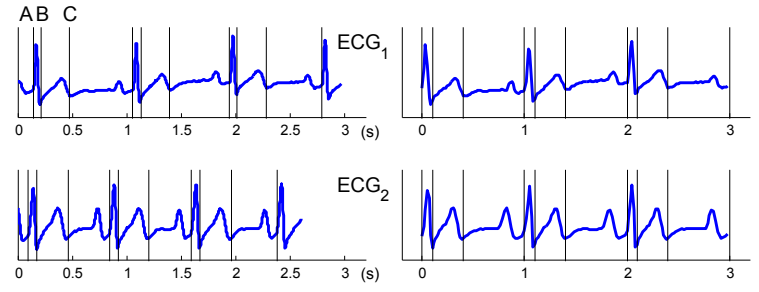

Figure 2: Temporal synchronization of two patients with different heart rates (71 bpm and $80 \mathrm{bpm}$, respectively), and different dynamics within their cardiac cycles (A: onset of QRS, B: aortic valve opening, C: aortic valve closure). Left: non-synchronized ECG, in seconds. Right: synchronized ECG, normalized timescale.

serves as a marker for the identification of the end of the isovolumic contraction (IVC) period, where $\mathrm{SF}$ is expected to be over. We used the absolute timing of ECG events proposed in the EchoPac software to locate these two events on the ECG associated to the studied sequence. This is done under the assumption that the timing between these events does not change between the sequences. This assumption is valid because the sequences have close heart rates, as they belong to the same session of acquisitions. In addition, in case of changes in heart rates, the diastolic period is mainly affected, while the timing of the events we chose is preserved as they belong to the systolic period. Manual corrections were performed in case of inaccurate timing proposed by the software.

Similar synchronization methods (Perperidis et al., 2005) identified a set of control points over sequences from MRI, but used image similarity. We preferred to rely on physiological information, as for US images the identification of these points using image data can be biased by respiratory or probe motion. In addition, the use of physiological events as temporal landmarks is believed to be more robust to pathology, as commented in Peyrat et al. (2009).

\subsection{Spatial normalization}

Spatial normalization consists in reorienting the computed velocity fields $\mathbf{v}^{k}(\mathbf{x}, t)$, initially defined according to the anatomy of patient $k$, to a reference anatomy used for local statistical comparison. We chose a simple strategy for spatial reorientation, which is illustrated in Fig. 3. It consists of four consecutive stages: defining a reference anatomy for the atlas, estimating mappings between every patient and the atlas at time $t=0$ (by convention, time $t=0$ was defined as the onset of the QRS complex), chaining paths to compute these mappings at time $t$, and reorienting the velocity fields $\mathbf{v}^{k}$ to the atlas anatomy at every time $t$ using these inter-series mappings.

Definition of a reference anatomy. The importance of using an average anatomy as reference in order to limit statistical bias has been commented in some publications about atlas construction (Guimond et al., 2000; Commowick and Warfield, 2009; Rueckert et al., 2003). In the case of atlases of shape, the distance between the compared shapes is defined from the mappings between the patients and the atlas. In our case, these mappings only serve for reorientation purposes, and do not directly intervene in the computation of a distance between patients. We therefore preferred to choose one series as reference for the sake of simplicity, under the assumption that the statistical bias on the $p$ value indexes used to quantify motion abnormality remains small.

The choice of a reference among the set of healthy volunteers was addressed using the group-wise normalized mutual information metric (GWNMI) proposed in Hoogendoorn et al. (2010), and criteria based on image quality (LV fully visible along the whole sequence, and low heart rate to achieve a higher temporal resolution of the atlas). The influence of such a reference choice is discussed in Sec. 4.4.5.

Mapping patients to the atlas at $t=0$. For every patient $k$, we compute the transformation $\varphi^{k \rightarrow r e f}(0)$, which maps the initial frame of this patient to the reference at time $t=0$. This mapping is estimated using diffeomorphic FFDs as in Sec. 2.1.

Aside from speckle noise, the visible anatomy differs in each sequence because of intrinsic character-

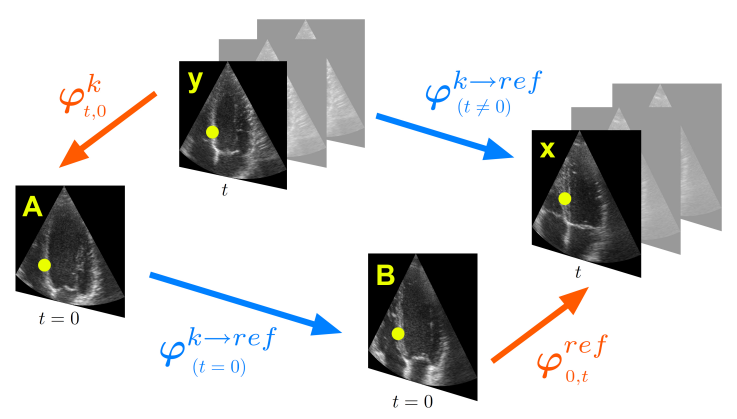

Figure 3: Illustration of the spatial reorientation at time $t$. 


\section{Preprint version accepted to appear in Medical Image Analysis. Final version of this paper will be available from http://www.sciencedirect.com/science/journal/13618415}

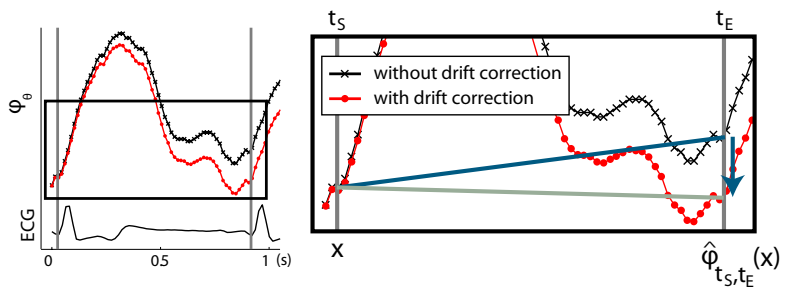

Figure 4: Illustration of the drift correction on one cycle. Black: tracking along the longitudinal direction without drift correction. Red: idem with drift correction.

istics of each patient (heart size and shape) and extrinsic parameters due to the US acquisition (probe orientation and US window size adapted to see the whole LV). As a consequence, we made the FFD registration start from a bulk affine transform. This step models rough differences common to the whole sequence, namely the ones due to US acquisition parameters and heart size.

Tracking the anatomy along sequences. Chaining the pairwise transformations defined in Sec. 2.1 allows to track the anatomy of each patient along the sequence. We obtain the transformations $\varphi_{0, t}^{k}$, which map the anatomy between times $t=0$ and $t$.

When chaining transformations resulting from registrations of consecutive frames, small errors accumulate, manifesting themselves as net drifts observed in the final myocardial point positions when computing full trajectories. These artifacts can be removed by applying to each point of the trajectory a correction ensuring that:

$$
\bigcirc_{t_{S} \leq t_{i}<t_{E}} \boldsymbol{\varphi}_{t_{i}, t_{i+1}}=\widehat{\boldsymbol{\varphi}}_{t_{S}, t_{E}}
$$

Here, $\bigcirc$ denotes the composition operator, $t_{S}$ and $t_{E}$ are the time-points starting two consecutive cardiac cycles, and $\widehat{\varphi}_{t_{S}, t_{E}}$ is the estimated transformation mapping frames at these time-points.

This correction is illustrated in Fig. 4. The transformation $\widehat{\varphi}_{t_{S}, t_{E}}$ is estimated using diffeomorphic FFDs as in Sec. 2.1, preceded by an affine registration step. It aims at taking into account probe motion during the acquisition, and adds robustness toward out-of-plane motion and filling variations between the different cardiac cycles, as the assumption $\widehat{\varphi}_{t_{S}, t_{E}}=\mathbf{I}$ generally made in other works (Ledesma-Carbayo et al., 2005) does not hold true in our database of $2 \mathrm{D}$ US sequences.
Mapping patients to the atlas at every time $t$. We estimate the transformations $\varphi^{k \rightarrow r e f}$ at time $t$ using the following chaining of transformations, which is illustrated in Fig. 3:

$$
\varphi^{k \rightarrow r e f}(t)=\varphi_{0, t}^{r e f} \circ \varphi^{k \rightarrow r e f}(0) \circ \varphi_{t, 0}^{k} .
$$

This strategy could later on be improved using the tools presented in Peyrat et al. (2009), in terms of robustness in the estimation of $\varphi^{k \rightarrow r e f}$ at every time $t$.

Reorientation to the reference. Reorientation of the velocity fields $\mathbf{v}^{k}$ is achieved at every point $(\mathbf{x}, t)$ using a push-forward action on vector fields ( $\mathrm{Tu}$, 2007):

$$
\mathcal{P}_{\phi}(\mathbf{v})=\left(\mathbf{D} \phi \circ \phi^{-1}\right) \cdot\left(\mathbf{v} \circ \phi^{-1}\right),
$$

where $\mathbf{v}=\mathbf{v}^{k}, \boldsymbol{\phi}=\boldsymbol{\varphi}^{k \rightarrow r e f}$ and $\mathbf{D}$ is the Jacobian operator. In Eq. 8, $\mathbf{D} \phi \circ \phi^{-1}$ represents the reorienting action on the vector fields moved to the new anatomical location by $\mathbf{v} \circ \boldsymbol{\phi}^{-1}$.

Reorientation of vector fields is illustrated in Fig. 5 and Fig. 6, which display the velocity field of one healthy subject before reorientation, i.e. directly over the anatomy of this subject, and after reorientation to the reference anatomy.

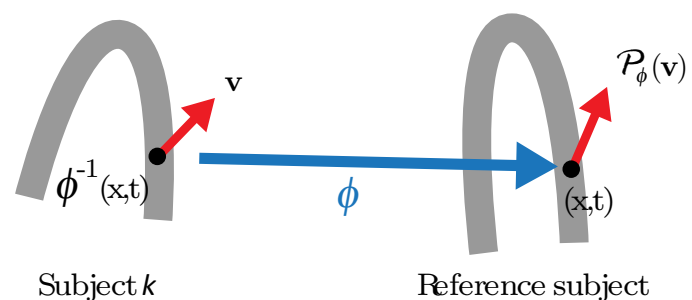

Figure 5: Illustration of the push-forward action on velocity fields at each location $(\mathbf{x}, t)$

\subsection{Statistics on velocities}

Velocities as defined in Sec. 2.2 belong to the tangent space of the group of diffeomorphisms. It means that because of the algebraic structure of the tangent space, classical statistics can be computed directly on the spatiotemporally normalized velocity fields, without the need of the log-Euclidean metrics described in Pennec and Fillard (2010).

We first compute their average and covariance to characterize the atlas population. Given $K$ different sample series $\left\{\mathcal{S}^{k} \mid k=1 \ldots K\right\}$, we obtain at 


\section{Preprint version accepted to appear in Medical Image Analysis. Final version of this paper will be available from http://www.sciencedirect.com/science/journal/13618415}
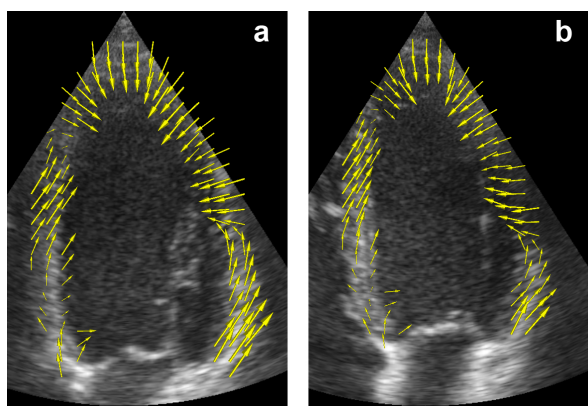

Figure 6: Velocity field $\mathbf{v}^{k}$ over the anatomy of subject $k(a)$ and after reorientation to the anatomy of subject ref $(b)$. Images correspond to the LV region during systole. Arrows have been scaled for optimal visibility.

any desired point $(\mathbf{x}, t)$ the average $\overline{\mathbf{v}}$ and the covariance matrix $\boldsymbol{\Sigma}_{\mathbf{v}}$ from the set of velocities $\mathbf{v}^{k}$, defined as:

$$
\overline{\mathbf{v}}=\frac{1}{K} \sum_{k=1}^{K} \mathbf{v}^{k} \quad \text { and } \quad \boldsymbol{\Sigma}_{\mathbf{v}}=\frac{1}{K-1} \mathbf{V}^{t} \cdot \mathbf{V}
$$

Here $\mathbf{V}^{t}=\left[\left(\mathbf{v}^{1}-\overline{\mathbf{v}}\right)|\ldots|\left(\mathbf{v}^{K}-\overline{\mathbf{v}}\right)\right]$ is the $M \times K$ matrix whose columns are the centered velocity samples at $(\mathbf{x}, t)$ and $M$ is the dimensionality of the data. In our case, $M=2$ (2D US).

Then, we use the atlas for the comparison of the velocities of a given patient to the population used for its construction. We chose Hotelling's $T$-square statistic (Hotelling, 1931) to perform abnormality tests on multivariate data, which is equivalent to the Mahalanobis distance in the particular case where a single sample is compared to a population:

$$
\tau^{2}=\alpha(\mathbf{v}-\overline{\mathbf{v}})^{t} \cdot \boldsymbol{\Sigma}_{\mathbf{v}}^{-1} \cdot(\mathbf{v}-\overline{\mathbf{v}}),
$$

where $\alpha=K /(K+1), \mathbf{v}$ is the velocity to compare to the atlas, and $\overline{\mathbf{v}}$ and $\boldsymbol{\Sigma}_{\mathbf{v}}$ are the previously described average and covariance matrix computed for the population atlas.

We use the $p$-value obtained from the Hotelling's $T$-test as quantitative index assessing abnormality. The $p$-value is computed from the cumulative function associated to the studied statistical distribution. This computation is performed under the assumption that the local distribution of myocardial velocities within the atlas population is gaussian. This assumption is justified in Sec. 4.3.

Leave-one-out cross-validation is used to compute the $p$-values within the atlas population.
In the following sections, we apply the previously described framework to build a statistical atlas of motion from a population of healthy subjects. We then use the atlas for the individual comparison of CRT patients to the atlas population chosen as reference, using the tools described in Sec. 3.3.

\section{Validation on 2D US image sequences}

In this section, the atlas construction steps are validated in terms of registration accuracy and reproducibility of the spatiotemporal alignment scheme. Special attention is paid to the quality of the atlas population (number of subjects, statistical distribution, chosen reference, and temporal resolution compared to the population of CRT patientss).

\subsection{Patient population and data acquisition}

Two-dimensional echocardiographic image sequences were acquired in an apical 4-chamber view for two populations of subjects, using a GE Vivid 7 echographic system (GE Vingmed Ultrasound A.S., Horten, Norway). The choice of the apical 4-chamber view is led by the fact that it is the one used in clinical routine for the assessment of the fast SF pattern. The atlas of normal velocities was constructed from 21 healthy volunteers (age $30 \pm 5$ years, 14 male). The patient population studied included 14 patients (age $67 \pm 8$ years, 8 male) that were candidates for CRT based on current clinical guidelines (symptomatic heart failure with long QRS length and low ejection fraction) and that visually had abnormal septal motion on a transthoracic echocardiographic examination. The study protocol was approved by the Hospital Clínic (Barcelona, Spain) ethics committee and written informed consent was obtained from all patients.

Physiological differences between patients constrain the acquisition parameters, which will differ in terms of temporal resolution and image quality. Images were acquired during breath-hold to minimize the influence of respiratory motion. Resolution was optimized during the acquisition of healthy subjects' sequences, and corresponds to an average frame rate of $60 \mathrm{fps}$ and a pixel size of $0.24 \times 0.24 \mathrm{~mm}^{2}$. The CRT patients involved in this study have dilated hearts compared to the healthy population. Thus, they require the use of a broader US sector so that the whole LV is still covered by the US beam. The temporal resolution of the sequences is thus lower for these patients due to this 


\section{Preprint version accepted to appear in Medical Image Analysis. Final version of this paper will be available from http://www.sciencedirect.com/science/journal/13618415}

constraint (around half the frame rate). Their average pixel size is $0.29 \times 0.29 \mathrm{~mm}^{2}$.

\subsection{Tools for visualizing spatiotemporal abnormal- ities}

The statistical tools described in Sec. 3.3 return a $p$-value index at every location $(\mathbf{x}, t)$, which can be visualized with the following tools, depending on the type of application targeted. Decoupling the spatial and temporal dimensions is particularly adapted for a precise localization of any motion abnormality (Sec. 5.1). In the following sections, another convenient mode of representation is used to visualize abnormalities in both spatiotemporal dimensions at the same time. In such maps, the horizontal axis represents time and the position in the septum (basal inferoseptal [BI], mid inferoseptal [MI], and apical septal [AS]) is used as vertical axis (right part of Fig. 7). The representation of the $p$-value in this space is similar to anatomical M-mode echocardiographic images, classically used to visualize wall motion over time. In order to highlight the inward and outward events of SF, in comparison with other patterns of abnormal motion of the septum (Sec. 5.3), the color-code used in these maps encodes the $p$-value in a logarithmic scale, multiplied by the sign of the radial velocity. Blue color represents highly abnormal inward motion of the septum, red color representing highly abnormal outward motion. The definition of local longitudinal and radial directions is illustrated in the left part of Fig.7.
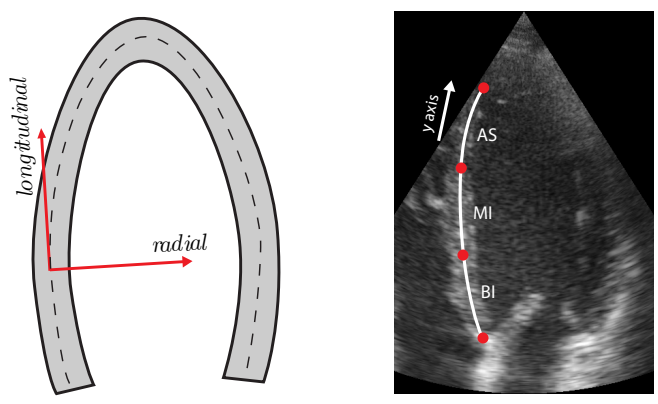

Figure 7: Left: Local representation of radial and longitudinal, defined as orthogonal and tangential to the septum medial line (dashed line), respectively. Right: Representation of the septal segments visible in the 4-chamber view (basal inferoseptal [BI], mid inferoseptal [MI], and apical septal [AS]) and used as vertical axis in the spatiotemporal maps of abnormality.

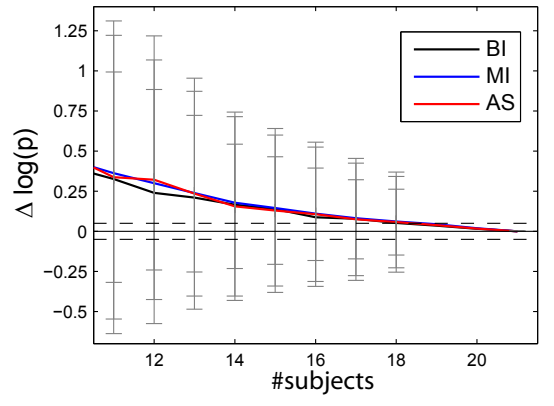

\begin{tabular}{c|ccc} 
Segment & IVC $^{*}$ & Systole $\backslash$ IVC $^{\dagger}$ & Diastole \\
\hline BI & $17.0 \pm 1.6$ & $16.8 \pm 1.2$ & $17.9 \pm 1.3$ \\
MI & $16.7 \pm 1.0$ & $17.1 \pm 1.5$ & $18.0 \pm 1.1$ \\
AS & $17.9 \pm 1.5$ & $19.3 \pm 1.5$ & $19.4 \pm 1.6$
\end{tabular}

* Isovolumic contraction

$\dagger$ Systole excluding the IVC period

Figure 8: Top: Normalized evolution of the motion abnormality indexes of one CRT candidate, versus the size of the atlas population. Average over the cardiac cycle and the septal segments (basal inferoseptal [BI], mid inferoseptal [MI], and apical septal $[\mathrm{AS}])$. Error bars represent the standard deviation over 100 random combinations of $K_{s}<K$ subjects. Bottom: values above which this evolution stabilizes to its final value $\pm 5 \%$ (dashed line), per cardiac segment and temporal window of the cardiac cycle. Average \pm standard deviation values over the set of 14 CRT candidates.

\subsection{Relevance of the atlas population}

The computation of a distance to normality assumes that the atlas population is representative of normality. In this study, the atlas population has non-dilated hearts, no cardiac dysfunction, and its baseline characteristics (QRS width, LV volumes and ejection fraction) match with the values found in the literature for a population of patients with normal cardiac function (Feigenbaum, 1994).

Number of subjects. To justify that the statistics are not biased due to the number of subjects in the atlas population $(K=21)$, we computed the evolution of the motion abnormality index ( $p$-value) for an atlas population made of $K_{s}<K$ subjects. This experiment is summarized in Fig. 8, in which the indexes were computed for a reduced set of 14 CRT candidates at each spatiotemporal location $(\mathbf{x}, t)$. These values were normalized towards the value obtained for the largest atlas population, so that the evolution is represented in the same magnitude scale (\%). The plot on the top represents this evolution for the three septal segments of one CRT candidate. For each value of $K_{s}<K$, the experiment 


\section{Preprint version accepted to appear in Medical Image Analysis. Final version of this paper will be available from http://www.sciencedirect.com/science/journal/13618415}

\begin{tabular}{cccc}
$(\%)$ & Segment & SW & LF \\
\hline \multirow{3}{*}{$\mathbf{v}_{1}$} & BI & $93.5 \pm 4.2$ & $14.4 \pm 4.0$ \\
& MI & $92.8 \pm 4.7$ & $15.0 \pm 3.8$ \\
& AS & $92.9 \pm 4.9$ & $14.7 \pm 4.0$ \\
\hline \multirow{2}{*}{$\mathbf{v}_{2}$} & BI & $88.7 \pm 8.3$ & $17.7 \pm 5.0$ \\
& MI & $89.6 \pm 7.7$ & $17.1 \pm 5.4$ \\
\hline \multicolumn{2}{r}{$\operatorname{randn}(21,10000)$} & $86.9 \pm 10.1$ & $18.6 \pm 6.0$ \\
\hline
\end{tabular}

Table 2: Shapiro-Wilk (SW) and Lilliefors (LF) tests for the distribution of myocardial velocities from 21 healthy volunteers, at each septal segment. The components of velocities along each eigendirection $\left(\mathbf{v}_{1}\right.$ and $\left.\mathbf{v}_{2}\right)$ were treated independently. Bottom line: generation of 21 normally distributed random numbers, repeated 10000 times.

was repeated for 100 random combinations of $K_{s}$ subjects (vertical error bars). In each spatiotemporal region, the number of subjects above which this evolution stabilizes to its final value $\pm 5 \%$ is summarized in the table of Fig. 8 (average \pm standard deviation over the set of 14 CRT candidates). Based on these values, we can reasonably trust an atlas built with all the available healthy volunteers (21 subjects).

Statistical distribution assumptions. We computed the Shapiro-Wilk and the Lilliefors tests (Shapiro and Wilk, 1965; Lilliefors, 1967) at each location $(\mathbf{x}, t)$ to check the gaussianity of the local distribution of the atlas velocities, as assumed for the computation of local $p$-values. The results are summarized in Tab. 2, which shows the average values and standard deviation of these tests over the three septal segments, along each eigendirection of the velocity distribution, independently. The last line presents the values of these tests for the generation of 21 normally distributed random numbers, repeated 10000 times. Based on these values, we can reasonably consider that the distribution of velocities is gaussian at each point $(\mathbf{x}, t)$.

\subsection{Validation of the atlas construction steps \\ 4.4.1. Intra-sequence registration accuracy}

We first evaluated the quality of our intrasequence registration by comparing it to manual landmarking. Three observers manually segmented the endocardium border of the septal wall for the whole set of subjects (volunteers and CRT candidates), at four temporal instants: onset of $\mathrm{QRS}$, AVO, AVC and onset of QRS for the subsequent cycle. For each observer, the shape delineated at the first of these instants was then propagated along the whole cycle using the displacement fields computed by our registration algorithm. Finally, its position at the three remaining instants was compared to the delineation made by the observer at these instants. Intra- and inter-operator variability $\left(\delta_{\text {intra }}\right.$ and $\left.\delta_{\text {inter }}\right)$ were computed at each of the four instants listed above. For the intra-operator variability, each observer repeated the manual delineation ten times for one healthy volunteer, while inter-operator variability was obtained by comparing the delineations made by the three observers, for the whole set of subjects. We used a point-to-line distance for the comparison of the delineated curves and the propagated ones (average over the points of each septal segment). Table 1 presents the distance between the automatically propagated shapes and the delineation made by the observers, and compares it to the intra- and inter-observer variability. The intra-sequence tracking showed a precision comparable to the observers variability for all the instants. Lower accuracy is observed near the apex, due to the lower quality of the US images in this region, as commented in the discussion section of this paper.

\subsubsection{Inter-sequence registration accuracy}

The accuracy of the inter-sequence registration was evaluated in a similar fashion than described in

\begin{tabular}{|c|c|c|c|c|c|c|c|c|c|c|}
\hline \multirow{3}{*}{$\begin{array}{c}\text { Units } \\
\text { (pixels) }\end{array}$} & \multicolumn{6}{|c|}{ Intra-sequence } & \multicolumn{2}{|c|}{ Inter-sequence } & \multirow[b]{3}{*}{$\delta_{\text {intra }}$} & \multirow[b]{3}{*}{$\delta_{\text {inter }}$} \\
\hline & & VOL & & & CRT & & VOL & CRT & & \\
\hline & AVO & AVC & $\mathrm{Q}$ & AVO & $\mathrm{AVC}$ & Q & & & & \\
\hline $\mathrm{BI}$ & $5.7 \pm 1.8$ & $10.9 \pm 5.4$ & $5.0 \pm 3.5$ & $5.1 \pm 1.9$ & $7.2 \pm 3.6$ & $5.6 \pm 2.8$ & $5.6 \pm 2.8$ & $7.5 \pm 2.8$ & $2.7^{*}$ & $6.9 \pm 3.0$ \\
\hline MI & $4.3 \pm 1.4$ & $8.3 \pm 3.4$ & $4.3 \pm 3.0$ & $5.6 \pm 2.9$ & $4.9 \pm 2.4$ & $4.5 \pm 1.6$ & $5.6 \pm 3.1$ & $7.6 \pm 3.9$ & $2.2^{*}$ & $5.5 \pm 2.2$ \\
\hline AS & $6.7 \pm 3.6$ & $9.1 \pm 3.9$ & $5.4 \pm 2.8$ & $7.0 \pm 3.1$ & $7.4 \pm 3.2$ & $6.6 \pm 3.1$ & $8.5 \pm 2.7$ & $11.1 \pm 3.1$ & $2.9^{*}$ & $8.5 \pm 4.2$ \\
\hline
\end{tabular}

Table 1: Comparison of intra- and inter-sequence registration accuracy to the variability in manual delineation of the endocardial border. Intra-sequence: distance between shapes manually delineated at three instants of the cardiac cycle, and the shape delineated at the beginning of the cycle, propagated using the registration-based tracking along the sequence. Inter-sequence: distance between the shapes delineated at the onset of QRS of the studied cycle in each subject's anatomy, which were mapped to the reference, and the shape delineated in the reference anatomy. Results indicate the average \pm standard deviation over the whole set of volunteers and the whole set of CRT candidates, respectively. 


\section{Preprint version accepted to appear in Medical Image Analysis. Final version of this paper will be available from http://www.sciencedirect.com/science/journal/13618415}

VOL vs Atlas

Atlas $\approx 60 \mathrm{fps}$ VOL $\approx 30 \mathrm{fps}$
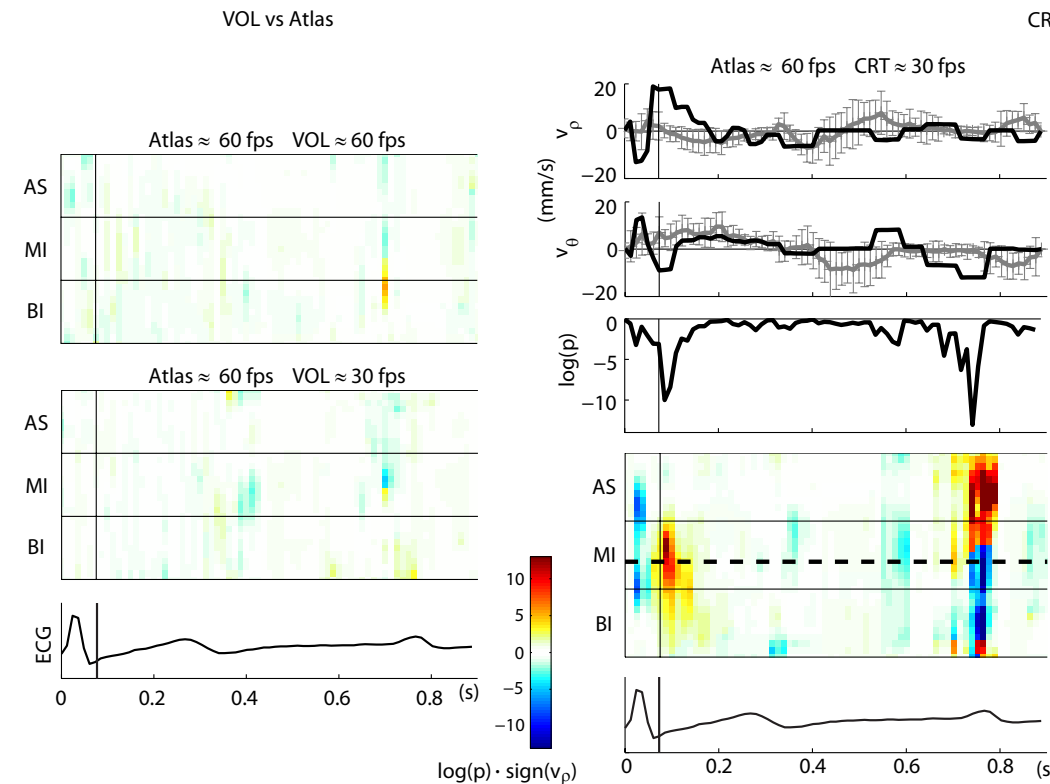

CRT vs Atlas
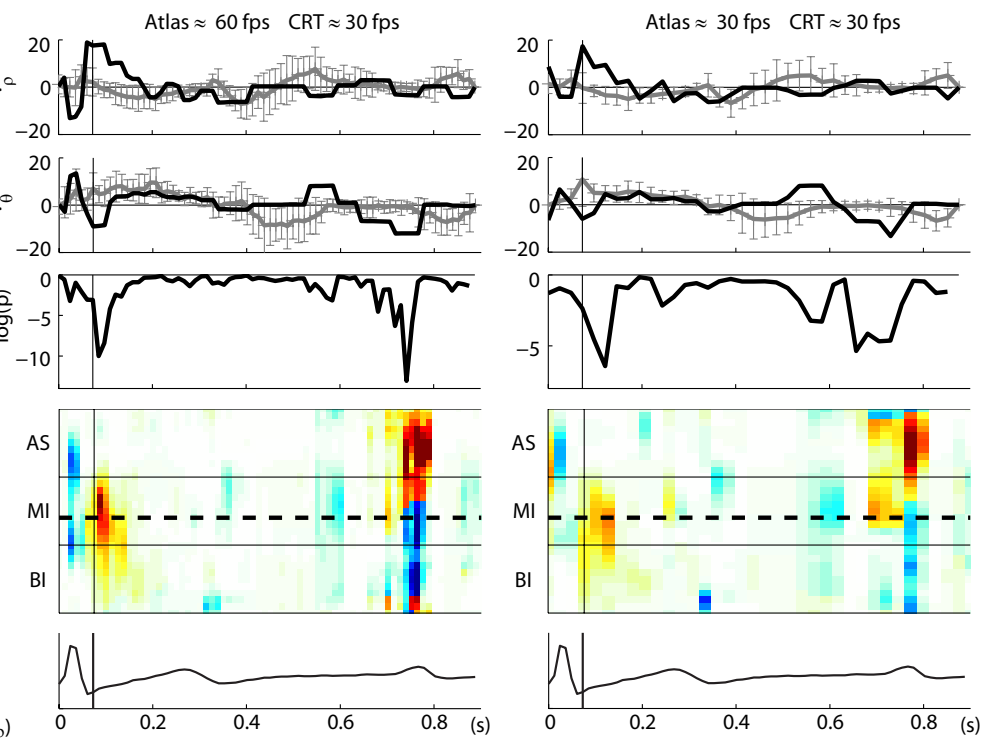

Figure 9: Left: Effect of a lower frame rate (volunteer to compare) on the $p$-value maps. Top: original frame rate (atlas $\approx 60$ fps, volunteer to compare $\approx 60 \mathrm{fps})$. Bottom: volunteer frame rate twice lower $(\approx 30 \mathrm{fps})$. Right: Effect of a lower frame rate (atlas population) on the $p$-value maps. First column: original frame rate (atlas $\approx 60$ fps, CRT candidates $\approx 30$ fps). Second column: atlas frame rate twice lower (atlas and CRT candidates $\approx 30 \mathrm{fps}$ ). Vertical line indicates the end of the IVC period.

Sec. 4.4.1 for the intra-sequence registration. For each subject, the shape delineated in the initial frame of the cycle was mapped to the reference anatomy using the transformation estimated by the inter-sequence registration. Then, the distance between the mapped shape and the shape delineated in the reference anatomy was used as an estimator of the inter-sequence registration accuracy. The experiment showed that inter-sequence registration precision is comparable to the observers variability.

\subsubsection{Influence of the temporal resolution}

In principle, differences in the temporal resolution of the atlas population and the set of CRT candidates could introduce bias on the abnormality measured. The two following experiments illustrate the influence of different frame rates on the computation of the $p$-value maps.

In the first experiment (left part of Fig. 9), a volunteer was compared to the atlas (using leaveone-out cross-correlation) at its original frame rate (around $60 \mathrm{fps}$ ) and at a reduced frame rate, obtained by using one frame out of every two in the volunteer's sequence. As can be inferred from Fig. 9, the two abnormality maps are very consistent with each other in spite of their large framerate differences: the pattern in both maps indicates low statistical support for abnormal motion. This confirms that the spots of motion abnormality observed on the $p$-value maps of the CRT candidates cannot just originate from the lower frame rate of these patients, compared to the atlas frame rate.

The second experiment illustrates the effect of a lower temporal resolution for the whole atlas population on the $p$-value maps. In the right part of Fig. 9, a CRT candidate with SF is compared to the atlas build with its original temporal resolution (around $60 \mathrm{fps}$, left column) and at a frame rate twice lower (right column). The figure shows that the localization of motion abnormalities is still feasible with an atlas built at a lower frame rate, but with seemingly less contrast and less resolution along the timescale.

\subsubsection{Overall synchronization}

In order to evaluate the quality of the spatiotemporal synchronization described in Sec. 3, we acquired four sequences for the same subject and checked that the estimated velocities overlapped after the synchronization to the reference spatiotemporal system of coordinates. A bad overlap would directly reflect artifacts introduced by the spatiotemporal synchronization. These sequences dif- 


\section{Preprint version accepted to appear in Medical Image Analysis. Final version of this paper will be available from http://www.sciencedirect.com/science/journal/13618415}

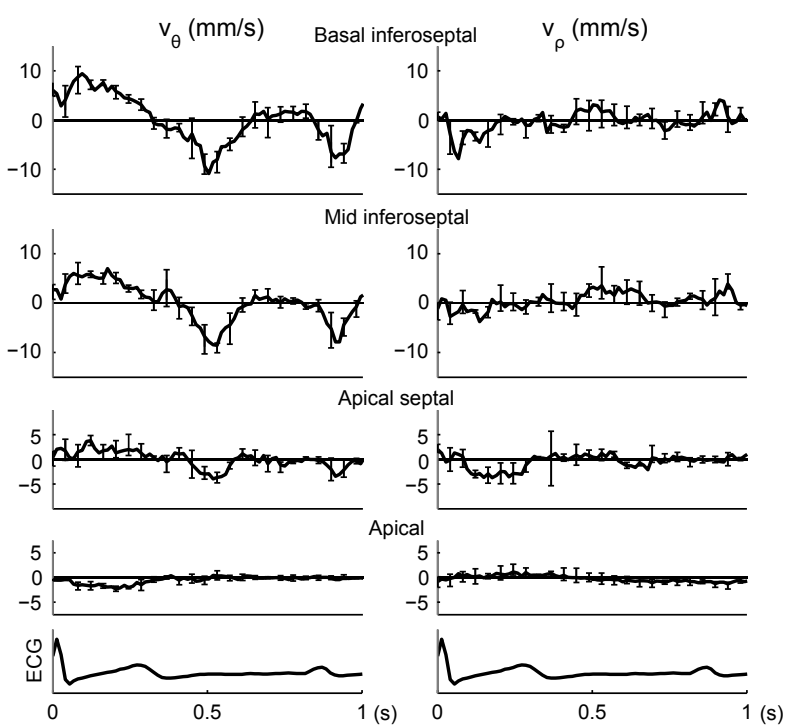

Figure 11: Repeatability in the normalization of velocities from four different acquisitions of the same subject, at four levels of the septum. Left: longitudinal velocities after reorientation; average \pm 1 standard deviation in the longitudinal direction. Right: idem with radial velocities. We only display one bar plot out of every three temporal instants for the sake of clarity. fer in terms of probe orientation and zoom of the US window, which were changed intentionally between the different acquisitions. They also differ in terms of heart rate, and have therefore different numbers of frames $(56,59,62$ and 64 frames for one cardiac cycle, respectively). In that way, the variability in the acquisition parameters is comparable to the one reached for the acquisition of different patients. Figure 11 illustrates the overlap between the velocities at four levels of the septum. The dispersion of the reoriented velocities (vertical bars) is measured in each direction from the corresponding diagonal coefficient of the covariance matrix $\Sigma_{\mathbf{v}}$, defined in Sec. 3.3. This dispersion reflects the accuracy of the spatiotemporal synchronization scheme, but may also result from differences in the myocardial velocities and the speckle patterns of the four acquisitions made, which could not be quantified with the imaging tools available for this study.

\subsubsection{Influence of the reference choice}

In order to understand the effects of the reference choice on the $p$-value maps, we repeated the atlas construction using different subjects as reference.
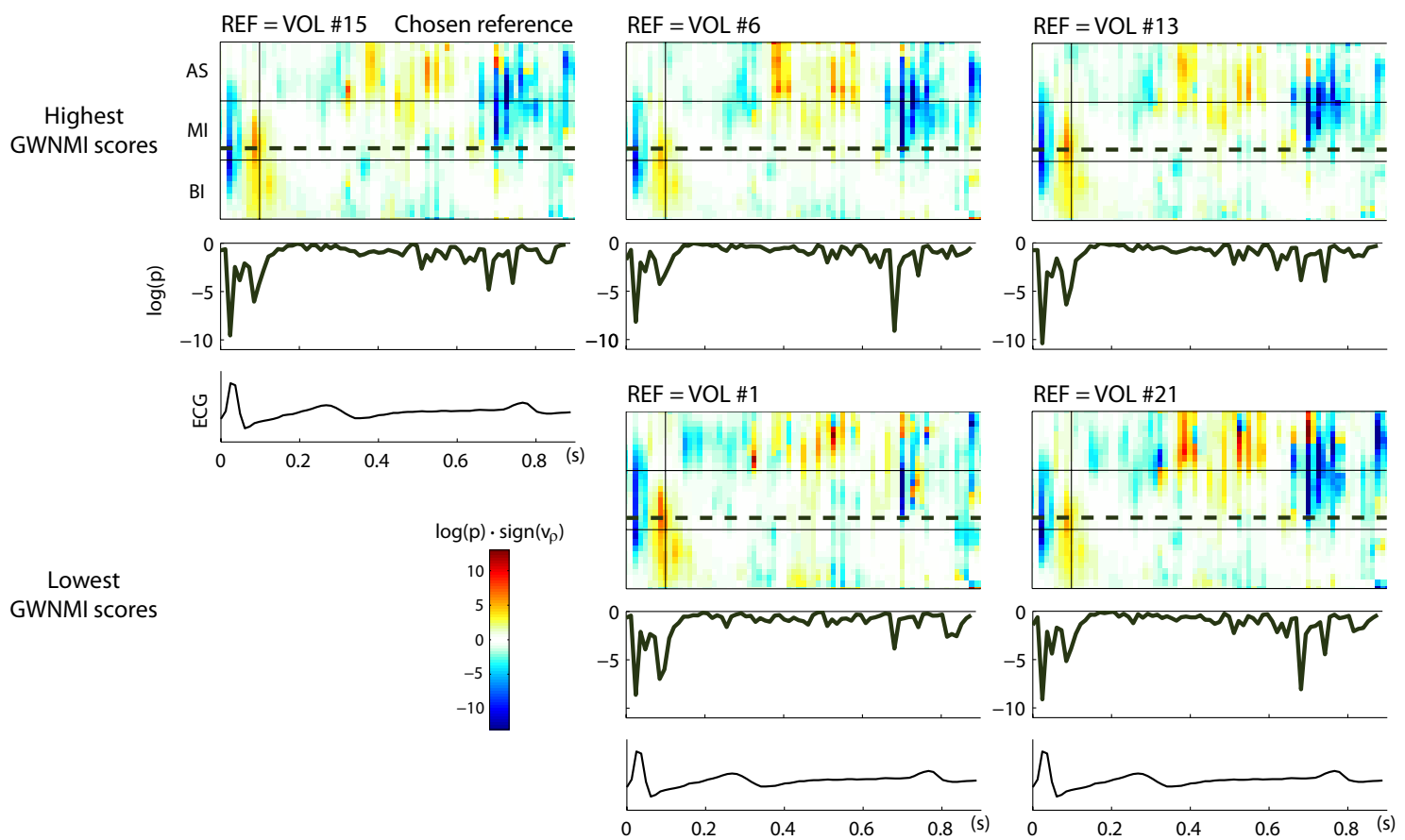

Figure 10: Influence of the reference choice on the atlas output ( $p$-value maps). VOL \#15 is the reference used in the rest of the paper, VOL \#6 and \#1 are the subjects with the two other best GWNMI scores, and VOL \#13 and \#21 the subjects with the two worst ones. 


\section{Preprint version accepted to appear in Medical Image Analysis. Final version of this paper will be available from http://www.sciencedirect.com/science/journal/13618415}
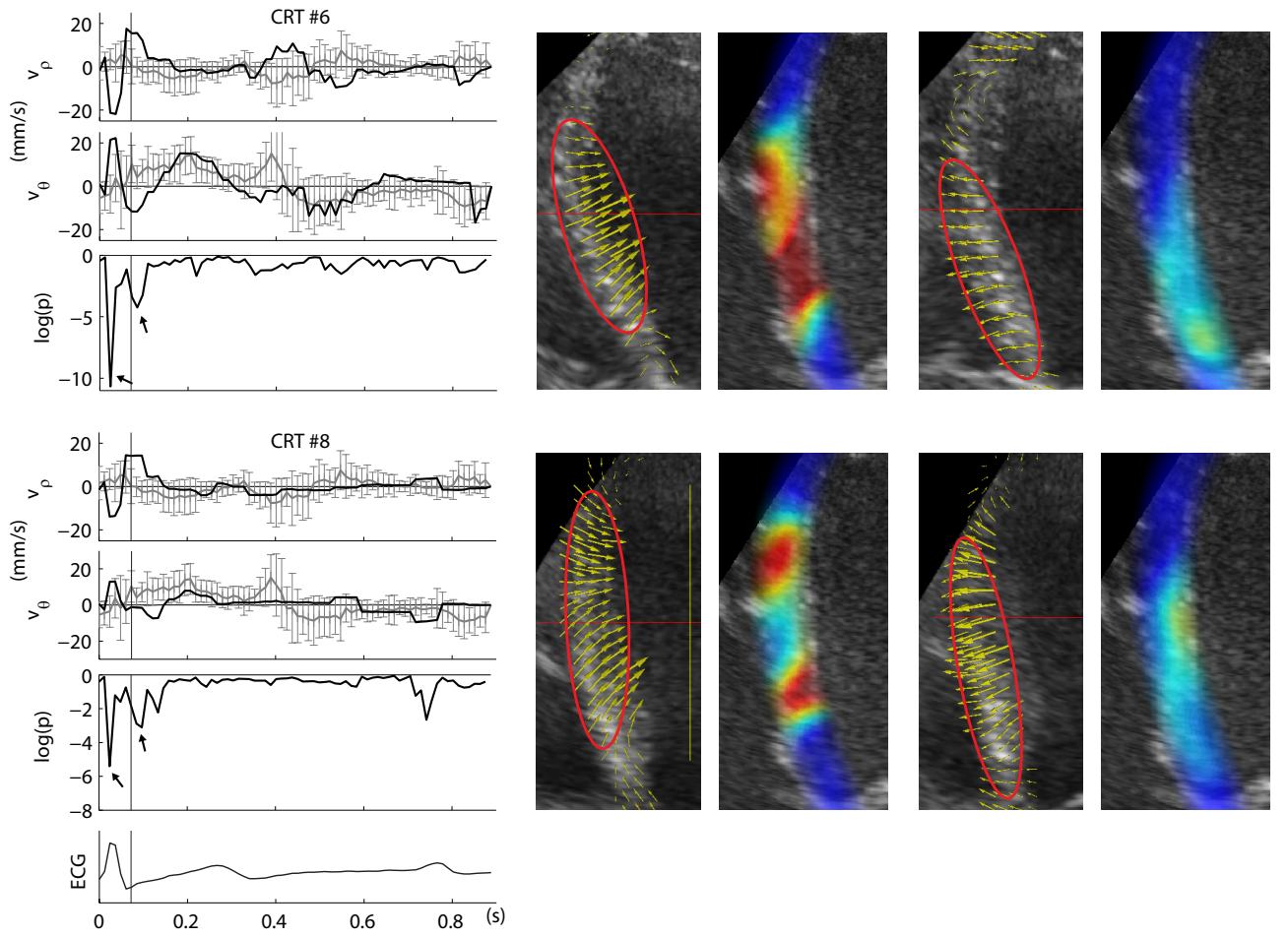

Figure 12: Comparison of two CRT patients with SF with respect to the atlas. Left: Temporal localization of the SF phenomenon, at the location where maximum excursion is observed (mid-inferoseptal level for both patients). From top to bottom: Radial velocities (gray: atlas average \pm 1 standard deviation in the radial direction, black: patient with SF), same plot with longitudinal velocities, and $p$-value plot along one cycle. Vertical line indicates the end of the IVC period. Arrows point out the inward and outward events. Right: Spatial localization of abnormality along the septum, at inward and outward events. In contrast, the LV of healthy subjects would mainly contract in the longitudinal direction. For each block: velocity field in the anatomy of patient $k$, and corresponding $p$-value map, defined in the reference anatomy. Arrows have been scaled by a global factor for optimal visibility. Warmer colors on the $p$-value maps indicate regions of higher abnormality.

We chose the subjects with the three best GWNMI scores (VOL \#15, which is the one used in the rest of the paper, \#6 and \#1), and the two worst ones (VOL \#13 and \#21). Few influence is observed on the $p$-value maps, as shown in Fig. 10 for CRT candidate \#6. This confirms the assumption introduced in Sec. 3.2, namely that the bias introduced by the use of another reference anatomy remains small.

\section{Application to the analysis of the CRT population}

The experiments described in this section demonstrate the performance of the proposed method for the accurate characterization of septal motion abnormalities, with particular attention paid to the SF mechanism. This characterization comprises a two-stage analysis: first, the localization of abnormal motion patterns in time and space (Sec. 5.1), then the interpretation of the observed patterns, which is done regionally focusing on the magnitude of the observed abnormalities (Sec. 5.2), and locally on $p$-value maps coupling the temporal and spatial dimensions (Sec. 5.3). The underlying objective of this section is to check whether the abnormality information obtained by our method is in agreement with the observations made by clinicians.

\subsection{Localization of motion abnormalities}

Temporal localization of septal flash. The left part of Fig. 12 illustrates the temporal analysis on two CRT patients presenting SF, at the location of the septum where maximal excursion is observed, including both velocity and $p$-value curves along one cardiac cycle. Low $p$-value means high degree of abnormality. Both plots exhibit a large abnormal inward velocity when the septum is activated, which is almost immediately followed by a fast outward 


\section{Preprint version accepted to appear in Medical Image Analysis. Final version of this paper will be available from http://www.sciencedirect.com/science/journal/13618415}

motion at the time when the infero-lateral wall contracts. This specific fast pattern, when occurring during the IVC period, determines the presence of SF, as described in Camara et al. (2009).

Spatial localization of septal flash. The $p$-value indexes obtained from our method directly allow a quantitative diagnosis at every point in space, as illustrated in the right part of Fig. 12. We display $p$-value maps at inward and outward events in order to analyze the way SF abnormality is distributed along the septum. For each block, we represent the initial velocity field in the anatomy of the studied patient, together with the corresponding $p$-value map, defined in the reference anatomy. This mode of representation illustrates the agreement in the location of SF between our abnormality maps (warmer colors) and the existing velocity fields (septum moves inward/outward, faster than the normal [higher magnitude of the velocities]. In contrast, healthy hearts would contract along the longitudinal direction).

\subsection{Accuracy in the quantification of abnormalities}

Three experts characterized the whole set of CRT candidates involved in the study, using analysis tools similar to those proposed in Parsai et al. (2009b). As a precise and objective localization using echographic tools is hard to reproduce, we asked the observers to make their diagnosis for three regions along the septum (basal inferoseptal, mid inferoseptal and apical septal). For each zone, they associated a score to the patient, among four possible values related to the degree of observed abnormality: 1 (no SF), 2 (uncertain), 3 (small SF), and 4 (large SF). For each zone of comparison, an agreement value between the observations from the different experts was obtained from the median value of their respective scores. The observed zone was marked as uncertain if the standard deviation between the different scores exceeded 1.

For each zone, we compared the previous observations to the motion abnormality indexes obtained from our analysis, as summarized in Fig. 13. For the patients with SF, the comparison was performed within the temporal window in which the inward and outward events occur, which were defined specifically for each patient, using the information on radial velocity $\mathbf{v}_{\rho}$ as follows:

$$
\begin{aligned}
\mathcal{I N} & =\left\{t \in I V C \mid \mathbf{v}_{\rho}(t)<0, t<\mathcal{O U} \mathcal{T}\right\} \\
\mathcal{O U} \mathcal{T} & =\left\{t \in I V C \mid \mathbf{v}_{\rho}(t)>0, t>\mathcal{I} \mathcal{N}\right\}
\end{aligned}
$$

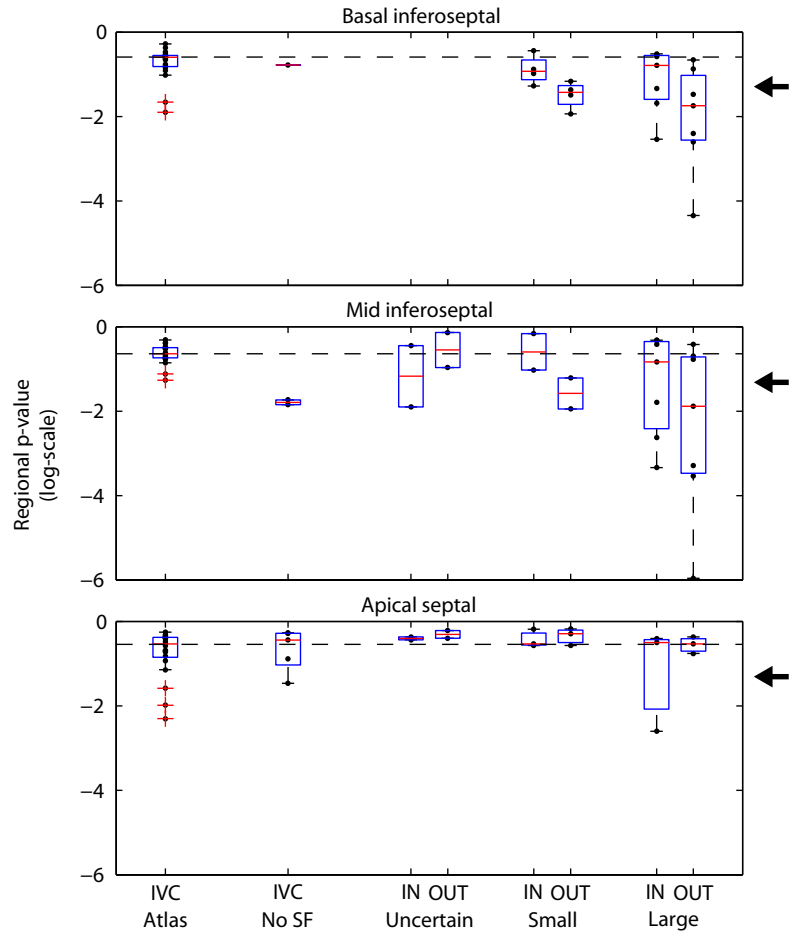

Figure 13: Local comparison between regional $p$-values and clinical diagnosis. Arrows on the right represent the value of 0.05 below which abnormality is considered significant. Dashed line indicates the median value of the atlas population.

The analysis was carried out on the whole IVC interval for the subjects with normal motion (atlas population) and for the patients without SF.

The diagnosis from the experts is only available regionally in time (within the temporal windows previously described) and space (the three regions along the septum). Thus, the comparison of their observations to the atlas-based quantification of abnormality was also done regionally. As the atlasbased $p$-values locally define a distance to normality, a representative $p$-value was computed for each region from their median over the spatiotemporal comparison zone.

A range for normality was obtained by including the atlas subjects in the analysis, for which $p$-values were obtained using leave-one-out cross-validation on the atlas population.

Figure 13 presents the comparison between the atlas-based diagnosis and the experts classification. In this figure, we observe the agreement between 


\section{Preprint version accepted to appear in Medical Image Analysis. Final version of this paper will be available from http://www.sciencedirect.com/science/journal/13618415}

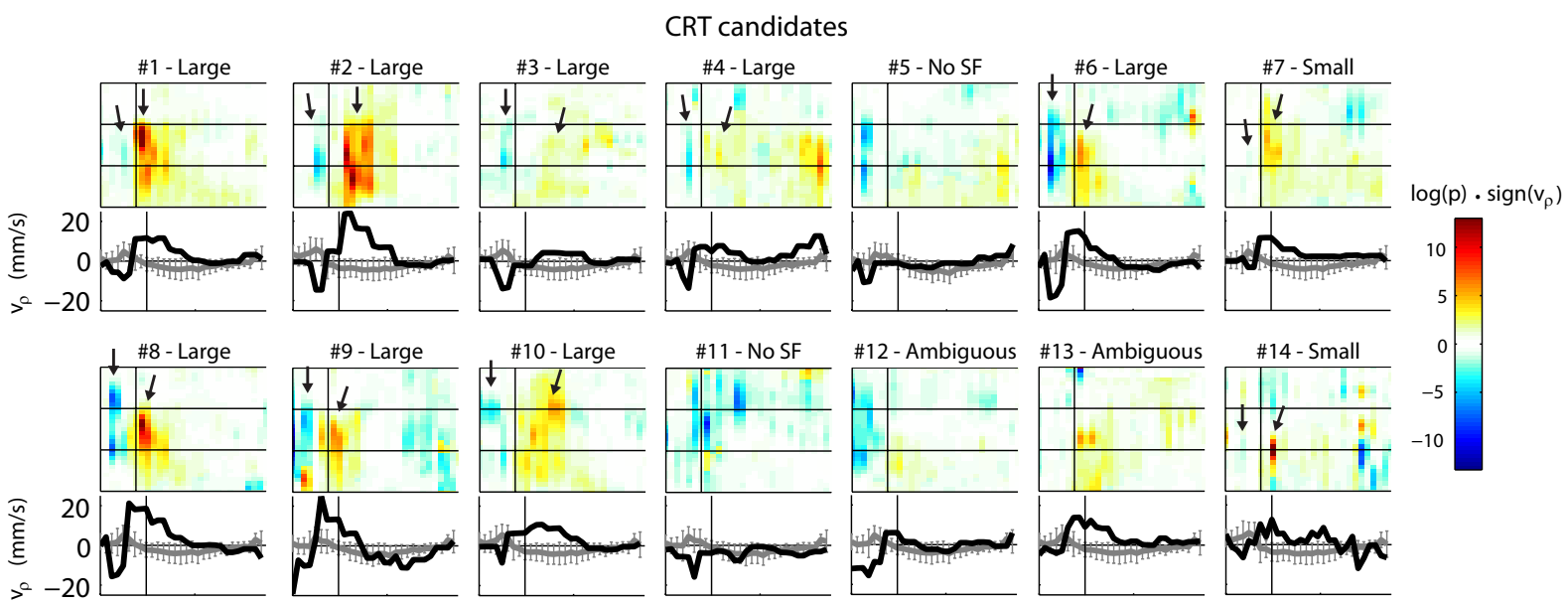

Figure 14: Motion abnormality maps and radial velocity profiles at the level of the septum with highest abnormality, during systole, for the whole set of CRT candidates. Black arrows point out the inward and outward motion during SF events, when present.

the comparison methods at the basal inferoseptal and mid inferoseptal levels. Indeed, significant abnormality (regional $p$-value $<$ median for the atlas) is observed in each group of patients, with noticeable differences depending on the grade of SF. This is mainly visible at the mid inferoseptal level, for which the septum has the highest amplitude of motion on the tested patients. In contrast, the whole atlas population lays in the normality range $(p$ value $<0.05)$. The different populations remain harder to distinguish at the apical septal level. The quality of the analysis in this region is commented in Sec. 6, together with the interpretation of the results for the zones for which the diagnosis was uncertain.

\subsection{How to differentiate between patterns: added- value of spatiotemporal maps of motion abnor- malities}

Combining both spatial and temporal quantification of motion abnormalities into a single map, as described in Sec. 4.2, facilitates the interpretation of the observed patterns and their comparison across patients. Figure 14 represents these abnormality maps for all the 14 CRT candidates, during the systole period. These maps are accompanied with a plot of the radial component of the velocity at the level of the septum with the highest motion abnormality for a better understanding of the observed abnormality patterns. The grade of SF obtained from experienced observers (Sec. 5.2) is in-

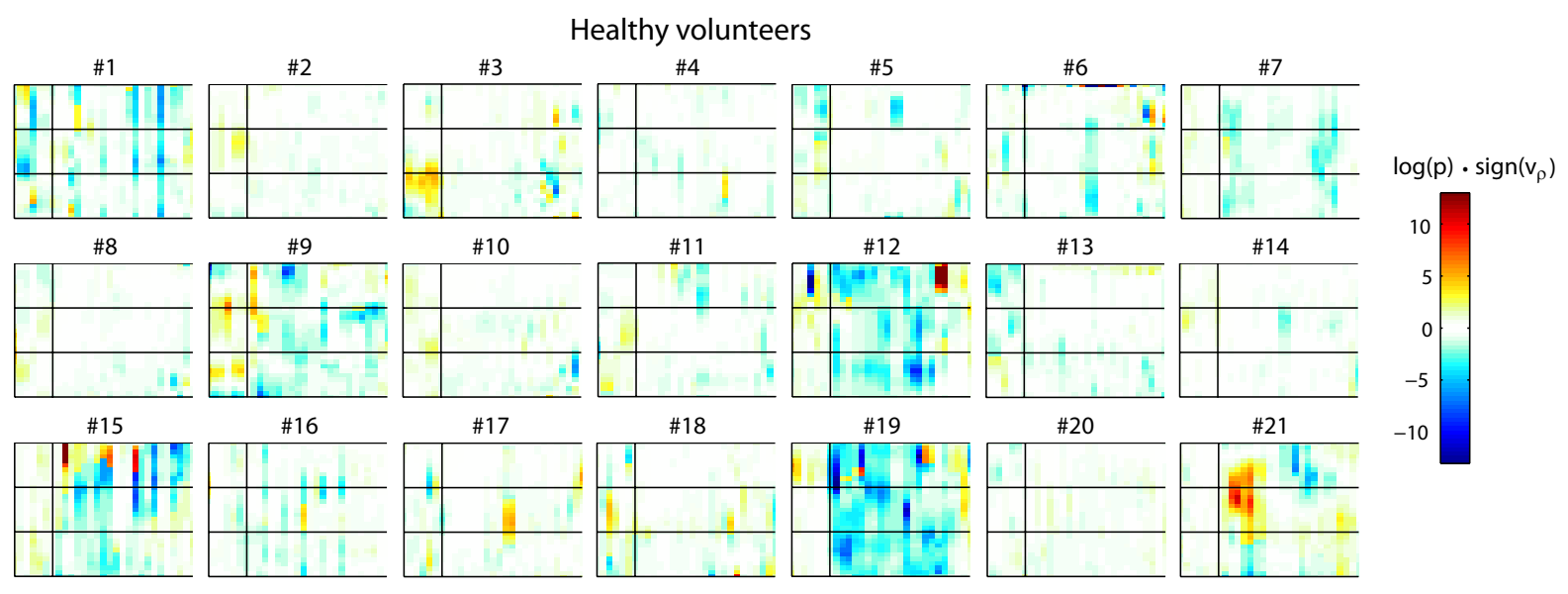

Figure 15: Motion abnormality maps during systole, for the set of volunteers. 


\section{Preprint version accepted to appear in Medical Image Analysis. Final version of this paper will be available from http://www.sciencedirect.com/science/journal/13618415}

dicated on the top. In this figure, a clear succession of inward (blue) and outward (red) abnormal motion starting during the IVC is visible on patients $\# 1, \# 2, \# 6$, \#8, \#9 and \#10, which were all diagnosed as "large SF" by the observers. Patients \#3 and \#4 were also diagnosed as "large SF," but the degree of motion abnormality is lower for both events. The inward motion pattern is almost absent for patient \#7, while both events are less visible for patient \#14. These two patients were diagnosed as "small SF." The SF pattern is absent in the remaining patients (\#5, \#11, \#12 and \#13), which were all categorized as "ambiguous SF" or "no SF." Patients \#5, \#11 and \#12 only show inward motion abnormalities. These patterns are interpreted in Sec. 6.

As a comparison, Fig. 15 represents these abnormality maps for the whole set of volunteers. Almost no abnormality is observed for most of these subjects. Volunteers for which abnormality is visible on these maps generally have higher velocities during the whole sequence, which is particularly noticeable on the radial velocity of \#12 and \#19. However, all these subjects belong to the atlas population, which means that these deviations from the average velocity profile are part of the atlas variance, and are therefore taken into account in the quantification of abnormalities for the set of CRT candidates.

\section{Discussion}

We have described a complete framework for the computation of a statistical atlas of motion, from its construction steps to the comparison of the atlasbased diagnosis to the observations made by experts. Our experiments demonstrate the feasibility of the proposed method on 2D US sequences. We first evaluated the quality of the atlas construction steps, and then demonstrated its applicability for an accurate localization of abnormal motion patterns, focusing on a specific pattern of the septum, namely SF.

The comparison tools illustrated in Fig. 12, 13, 14 and 15 shed light on the added value of the proposed indexes for the quantification of cardiac motion, in comparison with the tools currently used in clinical practice. By comparing patients within an atlas framework, we propose a local analysis of motion abnormalities, at every point in time and space (Fig. 12, 15 and 14) of a standardized anatomy. The use of our atlas-based indexes, which intrinsically embed a notion of normality, allows an accurate quantification of abnormality at every desired location. As illustrated in Fig. 13, our method agrees with the regional diagnosis performed by experts along the septum. In addition, it refines the information on the degree of abnormality observed and proposes some elements of interpretation for the zones where the diagnosis remained ambiguous.

In the case the subendocardium of the concerned region is infarcted, passive motion of the septal wall is observed when the lateral wall starts contracting and pushes the septum. Septal motion is therefore in the outward direction, but lasts longer than the IVC and is not a flash anymore. These patients are likely to belong to the left-right interaction class pointed out in Parsai et al. (2009b). In both cases, the observed zone will show lower abnormality (higher $p$-value) for the outward event, which is visible in particular in the plot of Fig. 13 representing the mid inferoseptal level, and in the maps of Fig. 14 for patients \#5, \#11 and \#12. A complementary analysis based on strain may help in discarding the ambiguities between true SF and infarcted zones with passive motion.

For clarity reasons, we preferred to set the focus of this paper on the construction of an atlas based on velocities, and the demonstration of the atlas performance in localizing and quantifying abnormalities in motion. Extension of the present method to strain measurements will be included in further work for a more complete characterization of the cardiac function, as recommended in Bijnens et al. (2009), and the assessment of other cardiac abnormalities.

Limitations. We chose to work with $2 \mathrm{D}$ US as it is the only modality used in clinical practice with sufficient temporal resolution to accurately identify fast motion patterns such as SF. However, the concepts developed in this paper could readily be applied to 3D US and other imaging modalities once the required temporal resolution is available in standard clinical acquisition protocols. The use of realtime 3D echocardiography (Soliman et al., 2009; De Craene et al., 2010) is particularly of interest to capture out-of-plane motion, which may increase the accuracy of the proposed analysis, and extend it to specific 3D motion patterns currently not captured by our method, such as torsion.

The quality of US images is however determinant for the relevance of the observations made in this 


\section{Preprint version accepted to appear in Medical Image Analysis. Final version of this paper will be available from http://www.sciencedirect.com/science/journal/13618415}

study. Depending on the tissue properties of each patient, the structure of the LV can be masked on some frames, especially at the apical level. Both the tracking accuracy and the clinical observations are affected, making the separation between the different populations less evident in this zone of the septum, as observed in Fig. 13.

\section{Conclusion}

In this paper, we proposed a new framework for the construction of an atlas that represents motion in a standard spatiotemporal coordinate system, and allows the comparison of patients against the atlas using quantitative indexes of abnormality. We evaluated the quality of the atlas construction steps, and illustrated the accuracy of the proposed indexes by applying the methodology to a population of healthy volunteers and CRT patients with left ventricular dyssynchrony. Our experimental results demonstrated the ability of the proposed method to quantify motion abnormalities at every location in time and space. The underlying objective was the characterization of the septal flash mechanism, which proved its interest for understanding response to CRT. Our pipeline could easily be extended to the quantification of abnormalities in strain for a more advanced characterization of the mechanisms influencing the response to CRT.

\section{Acknowledgments}

This research has been partially funded by the Industrial and Technological Development Center (CDTI) under the CENIT-CDTEAM and CENIT-cvREMOD programs and by the European Commission's project euHeart (FP7-ICT224495). Gemma Piella was supported by the Ramón y Cajal Programme from the Spanish Ministry of Science and Innovation. Adelina Doltra was supported by a Post-Residency Award from Fundació Clínic.

Arsigny, V., Commowick, O., Pennec, X., Ayache, N., 2006. A log-Euclidean framework for statistics on diffeomorphisms, in: Proc. MICCAI, LNCS 4190, pp. 924-931.

Ashburner, J., Csernansky, J.G., Davatzikos, C., Fox, N.C. Frisoni, G.B., Thompson, P.M., 2003. Computer-assisted imaging to assess brain structure in healthy and diseased brains. Lancet Neurology 2, 79-88.

Ashburner, J., Friston, K.J., 2000. Voxel-based morphometry - the methods. NeuroImage 11, 805-821.
Bax, J.J., Ansalone, G., Breithardt, O.A., Derumeaux, G., Leclercq, C., Schalij, M.J., Sogaard, P., St. John Sutton, M., Nihoyannopoulos, P., 2004. Echocardiographic evaluation of cardiac resynchronization therapy: ready for routine clinical use?: A critical appraisal. Journal of the American College of Cardiology 44, 1-9.

Bijnens, B.H., Cikes, M., Claus, P., Sutherland, G.R., 2009. Velocity and deformation imaging for the assessment of myocardial dysfunction. European Journal of Echocardiography 10, 216-226.

Byrd, R.H., Lu, P., Nocedal, J., Zhu, C., 1995. A limited memory algorithm for bound constrained optimization. SIAM Journal on Scientific Computing 16, 1190-1208.

Camara, O., Oeltze, S., De Craene, M., Sebastian, R., Silva, E., Tamborero, D., Mont, L., Sitges, M., Bijnens, B.H., Frangi, A.F., 2009. Cardiac motion estimation from intracardiac electrical mapping data: Identifying a septal flash in heart failure, in: Proc. FIMH, LNCS 5528, pp. $21-29$.

Chandrashekara, R., Mohiaddin, R., Rueckert, D., 2004. Analysis of 3-D myocardial motion in tagged MR images using nonrigid image registration. IEEE Transactions on Medical Imaging 23, 1245-1250.

Chandrashekara, R., Mohiaddin, R., Rueckert, D., 2005. Comparison of cardiac motion fields from tagged and untagged MR images using nonrigid registration, in: Proc. FIMH, LNCS 3504, pp. 425-433.

Cleland, J.G.F., Daubert, J.C., Erdmann, E., Freemantle, N., Gras, D., Kappenberger, L., Tavazzi, L., the Cardiac Resynchronization Heart Failure (CARE-HF) Study Investigators, 2005. The effect of cardiac resynchronization on morbidity and mortality in heart failure. New England Journal of Medicine 352, 1539-1549.

Commowick, O., Fillard, P., Clatz, O., Warfield, S.K., 2008. Detection of DTI white matter abnormalities in multiple sclerosis patients, in: Proc. MICCAI, LNCS 5241, pp. 975-982.

Commowick, O., Warfield, S.K., 2009. A continuous STAPLE for scalar, vector, and tensor images: An application to DTI analysis. IEEE Transactions on Medical Imaging 28, 838-846.

De Craene, M., Camara, O., Bijnens, B.H., Frangi, A.F., 2009. Large diffeomorphic FFD registration for motion and strain quantification from 3D-US sequences, in: Proc. FIMH, LNCS 5528, pp. 437-446.

De Craene, M., Piella, G., Duchateau, N., Silva, E., Doltra, A., D'Hooge, J., Camara, O., Sitges, M., Frangi, A.F., 2010. Temporal diffeomorphic free-form deformation for strain quantification in 3D-US images, in: Proc. MICCAI, LNCS. In press.

Delgado, V., Ypenburg, C., Van Bommel, R.J., Tops, L.F., Mollema, S.A., Marsan, N.A., Bleeker, G.B., Schalij, M.J., Bax, J.J., 2008. Assessment of left ventricular dyssynchrony by speckle tracking strain imaging: Comparison between longitudinal, circumferential, and radial strain in cardiac resynchronization therapy. Journal of the American College of Cardiology 51, 1944-1952.

Duchateau, N., De Craene, M., Silva, E., Sitges, M., Bijnens, B.H., Frangi, A.F., 2009. Septal flash assessment on CRT candidates based on statistical atlases of motion, in: Proc. MICCAI, LNCS 5762, pp. 759-766.

Durrleman, S., Pennec, X., Trouvé, A., Gerig, G., Ayache, N., 2009. Spatiotemporal atlas estimation for developmental delay detection in longitudinal datasets, in: Proc. MICCAI, LNCS 5761, pp. 297-304. 


\section{Preprint version accepted to appear in Medical Image Analysis. Final version of this paper will be available from http://www.sciencedirect.com/science/journal/13618415}

Feigenbaum, H., 1994. Echocardiography. Philadelphia: Lea and Febiger. chapter Echocardiographic measurements and normal values. pp. 658-695.

Fornwalt, B.K., Delfino, J.G., Sprague, W.W., Oshinski, J.N., 2009. It's time for a paradigm shift in the quantitative evaluation of left ventricular dyssynchrony. Journal of the American Society of Echocardiography 22, 672-676.

Grenander, U., Miller, M.I., 1998. Computational anatomy: an emerging discipline. Quarterly of Applied Mathematics LVI, 617-694.

Guimond, A., Meunier, J., Thirion, J.P., 2000. Average brain models: A convergence study. Computer Vision and Image Understanding 77, 192-210.

Hawkins, N.M., Petrie, M.C., MacDonald, M.R., Hogg, K.J., McMurray, J.J.V., 2006. Selecting patients for cardiac resynchronization therapy: electrical or mechanical dyssynchrony? European Heart Journal 27, 1270-1281.

Hoogendoorn, C., Whitmarsh, T., Duchateau, N., Sukno, F.M., De Craene, M., Frangi, A.F., 2010. A groupwise mutual information metric for cost efficient selection of a suitable reference in cardiac computational atlas construction, in: Proc. SPIE Medical Imaging 7623, 76231R.

Hotelling, H., 1931. The generalization of Student's ratio. The Annals of Mathematical Statistics 2, 360-378.

Khan, A.R., Beg, M.F., 2008. Representation of timevarying shapes in the large deformation diffeomorphic framework, in: Proc. IEEE ISBI, pp. 1521-1524.

Ledesma-Carbayo, M.J., Kybic, J., Desco, M., Santos, A., Sühling, M., Hunziker, P.R., Unser, M., 2005. Spatiotemporal nonrigid registration for ultrasound cardiac motion estimation. IEEE Transactions on Medical Imaging 24, 1113-1126.

Leporé, N., Brun, C., Chou, Y.Y., Chiang, M.C., Dutton, R.A., Hayashi, K.M., Luders, E., Lopez, O.L., Aizenstein, H., Toga, A.W., Becker, J.T., Thompson, P.M., 2008. Generalized tensor-based morphometry of HIV/AIDS using multivariate statistics on deformation tensors. IEEE Transactions on Medical Imaging 27, 129-141.

Lilliefors, H., 1967. On the kolmogorov-smirnov test for normality with mean and variance unknown. Journal of the American Statistical Association 62, 399-402.

Parsai, C., Baltabaeva, A., Anderson, L., Chaparro, M., Bijnens, B.H., Sutherland, G.R., 2009a. Low-dose dobutamine stress echo to quantify the degree of remodelling after cardiac resynchronization therapy. European Heart Journal 30, 950-958.

Parsai, C., Bijnens, B.H., Sutherland, G.R., Baltabaeva, A. Claus, P., Marciniak, M., Paul, V., Scheffer, M., Donal, E., Derumeaux, G., Anderson, L., 2009b. Toward understanding response to cardiac resynchronization therapy: left ventricular dyssynchrony is only one of multiple mechanisms. European Heart Journal 30, 940-949.

Pennec, X., Fillard, P., 2010. Statistical computing on nonlinear spaces for computational anatomy, in: Biomedical Image Analysis: Methodologies and Applications. Springer. In press.

Perperidis, D., Mohiaddin, R.H., Rueckert, D., 2005. Spatiotemporal free-form registration of cardiac MR image sequences. Medical Image Analysis 9, 441-456.

Petitjean, C., Rougon, N., Cluzel, P., Prêteux, F., Grenier, P., 2004. Quantification of myocardial function using tagged-MR and cine-MR images. International Journal of Cardiovascular Imaging 20, 497-508.

Peyrat, J.M., Delingette, H., Sermesant, M., Xu, C., Ayache, N., 2009. Registration of 4D cardiac CT sequences under trajectory constraints with multichannel diffeomorphic demons. IEEE Transactions on Medical Imaging In press.

Qiu, A., Albert, M., Younes, L., Miller, M.I., 2009. Time sequence diffeomorphic metric mapping and parallel transport track time-dependent shape changes. NeuroImage 45, S51-S60.

Rao, A., Chandrashekara, R., Sanchez-Ortiz, G.I., Mohiaddin, R., Aljabar, P., Hajnal, J.V., Puri, B.K., Rueckert, D., 2004. Spatial transformation of motion and deformation fields using nonrigid registration. IEEE Transactions on Medical Imaging 23, 1065-1076.

Rougon, N.F., Petitjean, C., Preteux, F.J., 2004. Building and using a statistical 3D motion atlas for analyzing myocardial contraction in MRI, in: Proc. SPIE Medical Imaging 5370, pp. 253-264.

Rueckert, D., Aljabar, P., Heckemann, R.A., Hajnal, J.V., Hammers, A., 2006. Diffeomorphic registration using Bsplines, in: Proc. MICCAI, LNCS 4191, pp. 702-709.

Rueckert, D., Frangi, A.F., Schnabel, J.A., 2003. Automatic construction of $3 \mathrm{D}$ statistical deformation models using non-rigid registration. IEEE Transactions on Medical Imaging 22, 1014-1025.

Rutz, A.K., Manka, R., Kozerke, S., Roas, S., Boesiger, P., Schwitter, J., 2009. Left ventricular dyssynchrony in patients with left bundle branch block and patients after myocardial infarction: integration of mechanics and viability by cardiac magnetic resonance. European Heart Journal 30, 2117-2127.

Shapiro, S.S., Wilk, M.B., 1965. An analysis of variance test for normality (complete samples). Biometrika 52, 591611.

Soliman, O.I., Geleijnse, M.L., Theuns, D.A., van Dalen, B.M., Vletter, W.B., Jordaens, L.J., Metawei, A.K., Al-Amin, A.M., ten Cate, F.J., 2009. Usefulness of left ventricular systolic dyssynchrony by real-time threedimensional echocardiography to predict long-term response to cardiac resynchronization therapy. American Journal of Cardiology 103, 1586-1591.

Sonne, C., Sugeng, L., Takeuchi, M., Weinert, L., Childers, R., Watanabe, N., Yoshida, K., Mor-Avi, V., Lang, R.M., 2009. Real-time 3-Dimensional echocardiographic assessment of left ventricular dyssynchrony: Pitfalls in patients with dilated cardiomyopathy. Journal of the American College of Cardiology - Cardiovascular Imaging 2, 802812 .

St John Sutton, M.G., Plappert, T., Abraham, W.T., Smith, A.L., DeLurgio, D.B., Leon, A.R., Loh, E., Kocovic, D.Z., Fisher, W.G., Ellestad, M., Messenger, J., Kruger, K., Hilpisch, K.E., Hill, M.R.S., for the Multicenter InSync Randomized Clinical Evaluation (MIRACLE) Study Group, 2003. Effect of cardiac resynchronization therapy on left ventricular size and function in chronic heart failure. Circulation 107, 1985-1990.

Stellbrink, C., Breithardt, O.A., Sinha, A.M., Hanrath, P., 2004. How to discriminate responders from nonresponders to cardiac resynchronisation therapy. European Heart Journal Supplements 6, 101-105.

Trouvé, A., 1998. Diffeomorphisms groups and pattern matching in image analysis. International Journal of Computer Vision 28, 213-221.

Tu, L.W., 2007. An Introduction to Manifolds. Springer. chapter 14 .

Voigt, J.U., 2009. Rocking will tell it. European Heart Journal 30, 885-886. 


\section{Preprint version accepted to appear in Medical Image Analysis. Final version of this paper will be available from http://www.sciencedirect.com/science/journal/13618415}

Worsley, K.J., Taylor, J.E., Tomaiuolo, F., Lerch, J., 2004. Unified univariate and multivariate random field theory. NeuroImage 23, S189-S195.

Young, A.A., Frangi, A.F., 2009. Computational cardiac atlases: from patient to population and back. Experimental Physiology 94, 578-596.

\section{Vitæ}

Acronyms.

CISTIB: Center for Computational Imaging \& Simulation Technologies in Biomedicine, Barcelona, Spain

UPF: Universitat Pompeu Fabra, Barcelona, Spain

Nicolas Duchateau received his Engineering degree in Optics from the Institut d'Optique, Palaiseau, France, in 2007, and his MSc degree in Mathematics, Vision and Machinelearning from the Ecole Normale Supérieure de Cachan, France, in 2008. He joined the CISTIB at the UPF in 2008 as $\mathrm{PhD}$ student to work under the supervision of Mathieu De Craene and Alejandro Frangi. His main research interests are in the use of image registration and statistical atlases for the quantification of heart motion and deformation.

Mathieu De Craene received his $\mathrm{PhD}$ degree from the Université catholique de Louvain, Belgium, in 2005. His thesis focused on developing automatic registration methods for medical images. He has been a visiting student at the Computational Radiology Laboratory, Boston, IL. He joined the CISTIB at the UPF in August 2006, where he works under the supervision of Alejandro Frangi. His main research interests are in the development of registration methods for the follow-up of endovascular treatment of cerebral aneurysms, and the quantification of heart motion and deformation.

Gemma Piella received her MSc degree in Telecommunication Engineering from the Universitat Politècnica de Catalunya (UPC), Barcelona, Spain, and her PhD degree from the University of Amsterdam, The Netherlands, in 2003. From 2003 to 2004, she was a visiting professor at the UPC. She then stayed at the Ecole Nationale des Telecommunications, Paris, France, as a postdoctoral fellow. She joined the UPF in 2005, now working at the CISTIB. Her main research interests are in the design of image registration techniques and their application to cardiac imaging.

Etelvino Silva received his degree in Telecommunications Engineering from the University of Valladolid, Spain, in 2005. Then, he started his PhD in Biomedical Engineering at the Universitat Politècnica de Catalunya (UPC), Barcelona, Spain, focusing on image post-processing in the context of cardiac resynchronization therapy. Since 2006, he has been working at the Cardiovascular Imaging Unit in Hospital Clínic, Universitat de Barcelona, Spain. His main research interests are in cardiac imaging, resynchronization therapy and ventricular tachycardia.

Adelina Doltra received her degree in Medicine from the Universitat Rovira i Virgili, Reus, Spain, in 2003. Since then, she has been working at Hospital Clínic, Barcelona, Spain, first as a resident in Cardiology and currently as a research fellow. She also spent a two-month period as an observer in Cardiac MRI and CT at Northwestern Memorial Hospital, Chicago, IL, in 2009. Her main research interests are in the contribution of cardiac imaging techniques in cardiac resynchronization therapy.

Marta Sitges received her degree in Medicine and Surgery from the Universitat Autònoma de Barcelona, Spain, in 1993, and her PhD degree from the Universitat de Barcelona, Spain, in 2003. Since 1993, she has stayed at the Hospital Clínic, Barcelona, Spain, as resident, research fellow and then as a permanent cardiologist. She also visited the Cardiovascular Imaging Center at the Cleveland Clinic Foundation, USA, during a one-year fellowship. Her main research interests are in cardiac imaging, resynchronization therapy, valve heart disease and cardiac remodeling.

Bart H. Bijnens received his MSc degree in Electronic Engineering and his $\mathrm{PhD}$ degree in Medical Sciences from the Catholic University of Leuven, Belgium. Since 1998, he is Associate Professor of Cardiovascular Imaging and Cardiac Dynamics at the Faculty of Medicine in Leuven. Since 2007, he is also Visiting Professor at the University of Zagreb, Croatia, where he resided for one year. From 2005 to 2006 he stayed at St George's Hospital in London, supervising clinical research. Since 2008, he is ICREA Research Professor at the Department of Information and Communication Technologies of the UPF, Barcelona Spain. His main research interests are in translational cardiovascular pathophysiology.

Alejandro F. Frangi received his MSc degree from the Universitat Politècnica de Cataluña, Barcelona, Spain, in 1996, and his $\mathrm{PhD}$ degree from the Image Sciences Institute, University Medical Center, Utrecht, NL, in 2001. He has been visiting researcher at Imperial College, London, UK, and in Philips Medical Systems BV, The Netherlands. $\mathrm{He}$ is currently Associate Professor at the UPF, ICREAAcademia Researcher, and leads the CISTIB group at the UPF. He is Senior Member of IEEE and Associate Editor of IEEE Transactions on Medical Imaging, Medical Image Analysis, the International Journal for Computational Vision and Biomechanics and Recent Patents in Biomedical Engineering journals. 